\title{
Adsorptive and Reductive Removal of Chlorophenol from Wastewater by Biomass-Derived Mesoporous Carbon-Supported Sulfide Nanoscale Zerovalent Iron
}

\author{
Hui Wang ${ }^{1}$, Sixiang Cai ${ }^{2, *}$, Liang Shan ${ }^{3}$, Min Zhuang ${ }^{1}$, Nan Li ${ }^{1}$, Guixiang Quan ${ }^{1}$ and \\ Jinlong Yan ${ }^{1, *}$ \\ 1 School of Environmental Science and Engineering, Key Laboratory for Advanced Technology in \\ Environmental Protection of Jiangsu Province, Yancheng Institute of Technology, Yancheng 224051, China; \\ whsl@ycit.cn (H.W.); z17802592726@163.com (M.Z.); ln_9597@163.com (N.L.); qgx@ycit.cn (G.Q.) \\ 2 State Key Laboratory of Marine Resource Utilization in South China Sea, Hainan University, \\ Haikou 570228, China \\ 3 Yancheng Environmental Engineering Technology Research and Development Center, School of \\ Environment, Tsinghua University, Yancheng 224051, China; shanliangsir@126.com \\ * Correspondence: 993049@hainu.edu.cn (S.C.); yjlyt@ycit.cn (J.Y.); Tel.: +86-515-8829-8805 (J.Y.)
}

Received: 1 December 2019; Accepted: 9 December 2019; Published: 16 December 2019

\begin{abstract}
Chlorinated compounds in a water environment pose serious threats to humanity. A nanoscale zerovalent iron (nZVI) has desirable properties for water dichlorination, but its reactivity is still limited by agglomeration and oxidation. In this study, the mesoporous carbon (MC) derived from biomass waste was prepared for immobilizing nZVI, and the nZVI@MC was further modified by sulfur (S-nZVI@MC) to relieve surface oxidation. The synergistic effect between nZVI and surface modification, the reaction conditions and the removal mechanism were investigated systematically. The characterization results showed nZVI was successfully loaded on the surface of MC, and the aggregation of nZVI was prevented. Moreover, sulfidation modification resulted in the formation of FeS on the surface of nZVI, which effectively alleviated surface oxidation of nZVI and promoted the electron transfer. Batch experiments demonstrated S-nZVI@MC had greatly enhanced reactivity towards 2,4,6-trichlorphenol (TCP) as compared to MC and nZVI, and the removal rate could reach $100 \%$, which was mainly attributed to the significant synergistic effect of MC immobilization and sulfidation modification. Furthermore, the TCP removal process was well described by a Langmuir adsorption model and pseudo-second-order model. The possible mechanism for enhanced removal of TCP is the fast adsorption onto S-nZVI@MC and effective reduction by S-nZVI. Therefore, with excellent reducing activity and antioxidation, S-nZVI@MC has the potential as a pollutant treatment.
\end{abstract}

Keywords: porous carbon; zerovalent iron; biomass; dichlorination; adsorption

\section{Introduction}

Nowadays, the scarcity of water resources and increasing water contamination are a worldwide environmental problem that have drawn extensive attention. Chlorophenols are a class of typical organic contaminants that have been utilized widely in various industrial and agricultural fields. Specifically, 2,4,6-trichlorphenol (TCP) has been listed among United States Environmental Protection Agency (US EPA) priority pollutants due to its high toxicity, mutagenicity, and carcinogenicity [1,2]. Various methods such as photodegradation, chemical reduction and adsorption have been developed to remove chlorophenols from contaminated water [3-5]. However, the methods usually involve high operating cost and complicated operation processes, which limit their practical application. Hence, it is highly desirable to develop a cost-effective method to remove TCP from contaminated water. 
In the past two decades, nanoscale zerovalent iron (nZVI) has been demonstrated to be an effective solution for the degradation/elimination of various pollutants including chlorinated organic compounds, heavy metal ions and antibiotics due to its strong reductive capability, low cost and environmental friendliness [6-8]. However, nZVI particles are easily agglomerated due to their high surface energy and intrinsic magnetic interaction, resulting in the rapid deactivation of $n Z V I$ in the operation process $[9,10]$. Moreover, nZVI particles are readily oxidized under ambient environmental conditions [11]. The above drawbacks limit the environmental application of nZVI.

Immobilizing nZVI particles on porous material supports has been demonstrated as a tactful way to obtain high-efficiency and stable nZVI [12]. On the one hand, porous supports have a certain adsorption capacity for the pollutants, thereby improving the removal efficiency. On the other hand, porous supports can provide stable sites for nZVI particles, and thus prevent the agglomeration of the nanoparticles. Compared with various porous materials, carbon supports have attracted great attention due to the large specific surface area, porous structure and good chemical stability $[13,14]$. For instance, Wu et al. found that the removal efficiency of bromate by nZVI/activated carbon can be improved due to its excellent adsorption performance [15]. Although the traditional activated carbon has a high specific surface area, micropores predominate in its structure, which is not conducive to the effective dispersion of nZVI, and the diffusion resistance also limits the transport of pollutant molecules. Therefore, mesoporous carbon has been applied for the nZVI support owing to the large surface area, the promotion of target pollutant diffusion as well as the excellent nZVI immobilization abilities provided by the mesoporous channels. Ling et al. found that loading nZVI on a mesoporous carbon significantly increased its reactivity towards nitrobenzene [16]. The mesoporous carbon is usually prepared through a templating method using mesoporous silica as hard templates or amphiphilic copolymer surfactants as soft templates and resin as carbon source. However, such fabricating approaches usually involve complicated and costly preparation procedures, which have limited their practical applications. Therefore, it is still challenging to synthesize mesoporous carbon-supported nZVI through a facile and economical method.

It should be noted that although supported nZVI can prevent the agglomeration and improve the stability of $\mathrm{nZVI}$, it is easily oxidized by water, $\mathrm{O}_{2}$ and other coexisting solutes, which would impede electron transfer from nZVI core to target contaminates [17]. In view of the above issue, recent research has found that sulfidation of nZVI is the most promising approach to suppress surface oxidation and increase reactivity of nZVI [18-20]. On the one hand, the iron sulfide shell on the surface of nZVI acts as a barrier to prevent nZVI contacting water and mitigating the its surface corrosion. On the other hand, the iron sulfide has higher electronegativity (5.02 V) than nZVI (4.04 V), which can facilitate the rapid transfer of electrons. For instance, Gao et al. found the reactivity of iron sulfide coated iron nanoparticles towards Cr(VI) was significantly better than bare nZVI [21]. He et al. demonstrated that the sulfidation can improve the dichlorination rate and selectivity of nZVI [22].

To take advantage of the recent development in $\mathrm{nZVI}$, a sulfidized nZVI supported on MC (S-nZVI@MC) was prepared and used to remove TCP from aqueous solutions. As seen from Figure 1, the MC was synthesized simply using straw waste as carbon source and colloidal silica as hard template, which simplified fabricating processes and reduced synthesis cost. With MC as a supporter, the S-nZVI was successfully loaded on its surface by reduction of Fe (III) ions with $\mathrm{NaBH}_{4}$ in the presence of dithionite. The aggregation and surface oxidation of nZVI has been effectively alleviated due to the synergistic effect of loading and sulfidation, which is beneficial to improve its reactivity and life. The S-nZVI@MC was further used for TCP removal from water and the effects of solution $\mathrm{pH}$, TCP initial concentration, S-nZVI@MC dosage were examined. The finding of this study paves the way to developing high-performance and low-cost nZVI for dichlorination and reduction of other pollutants. 


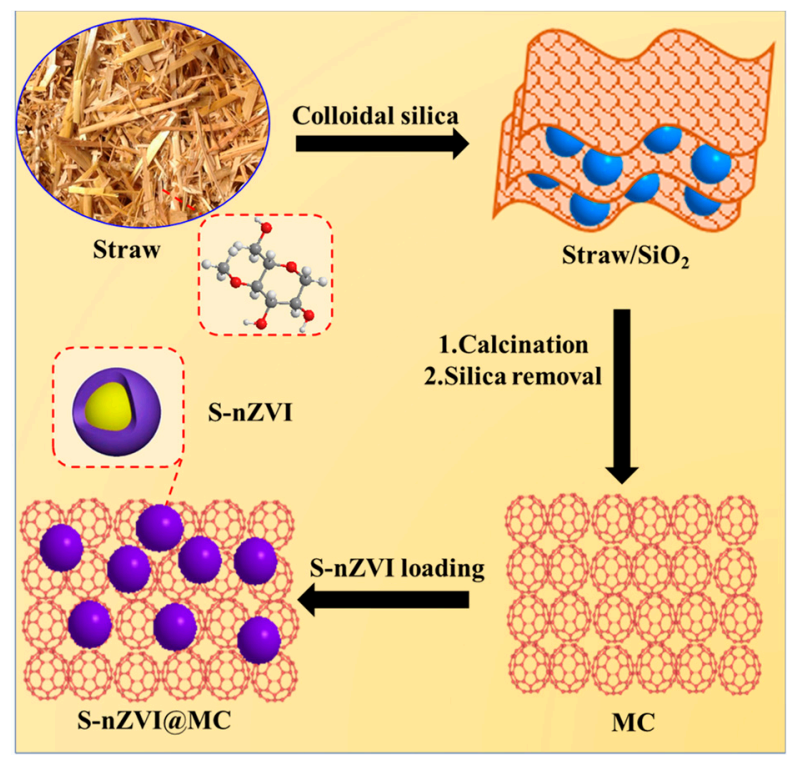

Figure 1. Schematic illustration of the sulfidized nanoscale zerovalent iron supported on mesoporous carbon (S-nZVI@MC) formation.

\section{Materials and Methods}

\subsection{Synthesis of S-nZVI@MC}

Materials: wheat straw was obtained from Yancheng, Jiangsu, P. R. of China. To remove impurities, the wheat straw is fully washed with deionized water and anhydrous ethanol before use. 2,4,6-Trichlorophenol (TCP, >98\%) was purchased from Aladding Chemistry Co. Ltd., Shanghai, China. Hydrofluoric acid (HF, AR) was obtained from Sinopharm Chemical Reagent Co., Ltd., Shanghai, China. Milli-Q water $\left(18.25 \mathrm{M} \Omega \mathrm{cm}^{-1}\right)$ was used throughout the experiment.

Per-carbonization of the wheat straw: in typical synthesis, wheat straw $(3.0 \mathrm{~g})$ was added into the $\mathrm{H}_{2} \mathrm{SO}_{4}$ solution $(100 \mathrm{~mL}, 5 \%, \mathrm{~V}: \mathrm{V})$, and then stirred for $15 \mathrm{~min}$. The above solution was transferred into the Teflon vessel and reacted for $12 \mathrm{~h}$ at $180^{\circ} \mathrm{C}$. After cooling to room temperature, the product was washed by deionized water until reaching a $\mathrm{pH}$ of 7 , and then dried at $80^{\circ} \mathrm{C}$ in a conventional oven. Finally, the per-carbonized wheat straw was obtained.

Synthesis of MC: typically, the per-carbonized wheat straw was soaked in $1.0 \mathrm{wt} \%$ colloidal silica aqueous solution, and mass ratio of silica/straw was 3:10. The above solution was evaporated at $85^{\circ} \mathrm{C}$ through continuous stirring until becoming a viscous solution. After drying at $80^{\circ} \mathrm{C}$ in a conventional oven, a solid powder was formed. To obtain the MC, the precursor was calcinated at $800{ }^{\circ} \mathrm{C}$ for $2 \mathrm{~h}$ under a $\mathrm{N}_{2}$ atmosphere with the ramp rate of $3^{\circ} \mathrm{C} / \mathrm{min}$. After $\mathrm{HF}$ etching and water wash, the silica can be removed and MC was finally formed.

Synthesis of S-nZVI@MC: $0.2 \mathrm{~g}$ MC was firstly added into $100 \mathrm{~mL}$ DI and ethanol mixture solution $(\mathrm{V}: \mathrm{V}=7: 3)$. After sonicating for 60-120 min, the above mixture solution was poured into a $250 \mathrm{~mL}$ three-necked bottom flask equipped with a mechanical stirrer, and a thermometer with a temperature controller and a $\mathrm{N}_{2}$ inlet; $1.15 \mathrm{~g}$ of $\mathrm{FeSO}_{4} \cdot 7 \mathrm{H}_{2} \mathrm{O}$ was then added to the three-neck flask, and after being stirred for about $20 \mathrm{~min}$ in $\mathrm{N}_{2}$ atmosphere, $100 \mathrm{~mL}$ containing $7.6 \mathrm{~g}$ sodium borohydride and different amounts of $\mathrm{Na}_{2} \mathrm{~S}_{2} \mathrm{O}_{6}$ (samples here referred as S-nZVI@MC-0.05, S-nZVI@MC-0.125, S-nZVI@MC-0.25, whose $\mathrm{S} / \mathrm{Fe}$ molar ratio were approximate $0.05,0.125$ and 0.25 , respectively) were added in a dropwise manner to the above $\mathrm{FeSO}_{4}$ solution. After the injection, stirring continued for $30 \mathrm{~min}$ to ensure adequate reaction. S-nZVI@MC were harvested by centrifugation and washed with ethanol three times and then dried in a vacuum. The obtained samples were carefully stored in the glove box filled with inert gas. 


\subsection{Characterization}

The detailed morphological information of the samples was further analyzed by transmission electron microscopy (TEM, JEOL JEM-200CX, Tokyo, Japan) and scanning electron microscopy (SEM, JEOL JEM-6700F, Tokyo, Japan) tests. Before the TEM testing, the samples were dispersed in ethanol via ultrasonication for $15 \mathrm{~min}$, and then deposited a few drops onto a carbon-coated copper grid. X-ray diffraction (XRD, Rigaku Corporation, Tokyo, Japan) measurements were recorded by the X-ray diffractometerusing $\mathrm{Cu} \mathrm{K} \alpha$ radiation $(40 \mathrm{kV}, 3020 \mathrm{~mA})$. X-ray photoelectron spectroscopy (XPS, Perkin-Elmer, Hopkinton, MA, USA) was tested on a Perkin-Elmer PHI 5000C ESCA (Waltham, MA United States) system with a dual X-ray source, using the $45 \mathrm{MgK \alpha}(1253.6 \mathrm{eV})$ anode and a hemispherical energy analyser. Before all tests, the samples were sealed into sample tubes in a glove box, and then shipped to the test instrument.

\subsection{Adsorptive and Reductive Performance}

Batch adsorption experiments were conducted by shaking a mixture of obtained S-nZVI@MC with $100 \mathrm{~mL}$ TCP aqueous solution of certain concentration in a series of $150 \mathrm{~mL}$ plastic bottles. Such adsorption experiment parameters as contact time (0-180 min), initial concentration of TCP (30-100 $\mathrm{mg} \mathrm{L}^{-1}$ ) and solution $\mathrm{pH}(4-11)$ were further investigated. The solution $\mathrm{pH}$ was adjusted by $0.1 \mathrm{M} \mathrm{HCl}$ or $\mathrm{NaOH}$ solution. The solid-liquid phases were taken out after shaking for a predetermined time, and followed by filtration using $0.22 \mu \mathrm{m}$ cellulose nitrate membrane filter. The concentration of TCP was measured with an ultraviolet-visible spectrophotometer (TU 1810, Beijing Purkinje General Instrument Co. Ltd., Beijing, China). The removal efficiency was calculated according to the following equations:

$$
\eta=\left(\mathrm{C}_{0}-\mathrm{C}_{\mathrm{t}}\right) / \mathrm{C}_{0}
$$

where $\mathrm{C}_{0}$ and $\mathrm{C}_{\mathrm{t}}\left(\mathrm{mg} \mathrm{L}^{-1}\right)$ are the initial and final concentrations of TCP in the feed solution, respectively.

\section{Results}

\subsection{Characterization}

The morphology of the MC and S-nZVI@MC was firstly investigated by TEM and SEM. As presented in Figure 2a,b the MC shows a well-formed porous structure, and the pore size is about $22 \mathrm{~nm}$, which is consistent with the size of the silica template. The mesoporous structure not only has a high specific surface area for nZVI particle dispersion, but also provides a large number of smooth channels for the TCP molecules diffusion. As shown in Figure 2c, the MC surface is covered with flake-like structure, indicating that the S-nZVI was successfully loaded on the MC. The S-nZVI has a core-shell structure, and the $\mathrm{Fe}^{0}$ core is loosely surrounded by FeS. The agglomeration of nZVI is obviously relieved, indicating the MC supporter can anchor nZVI and promote its dispersion. In addition, the FeS shell can also effectively reduce the magnetic attraction between nZVI particles and prevent its re-agglomeration. As seen from the SEM image of S-nZVI@MC in Figure 2d, the discrete S-nZVI with globular structure covered the surface of MC. Moreover, with elemental energy dispersive spectrometer (EDS)mapping shows a distribution of Fe, S, and C in S-nZVI @MC (Figure 2e-g). The notable Fe and $S$ distribution confirmed the presence of Fe and $S$ after the nZVI loading and sulfide modification. Therefore, combined with loading and sulfidation, the agglomeration of nZVI was effectively inhibited, which is beneficial to active site exposure, and thus promotes its reactivity. 


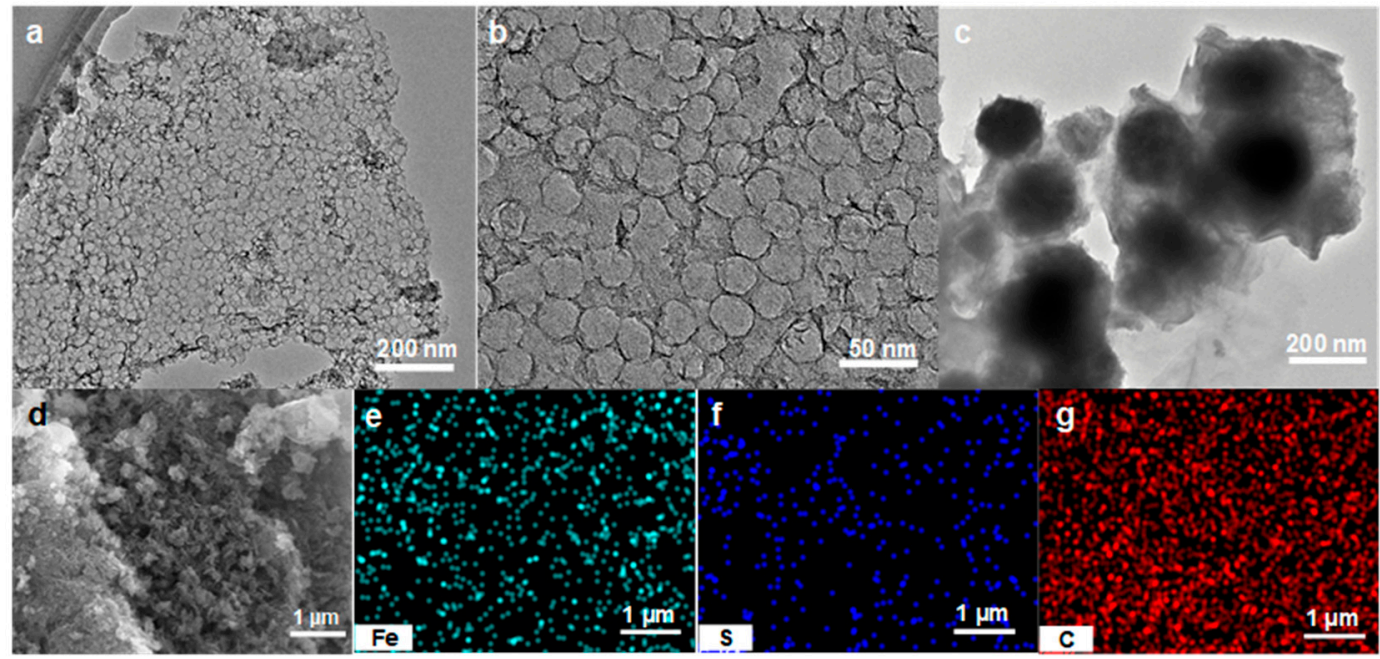

Figure 2. (a,b) Transmission electron microscope (TEM) images of mesoporous carbon (MC), (c) TEM image, (d) scanning electron microscope (SEM) image and (e-g) elemental mapping images of S-nZVI@MC.

Figure 3 shows the XRD patterns of S-nZVI@MC and nZVI. The pattern of MC shows two diffraction peaks at $2 \theta$ value of $23^{\circ}$ and $43^{\circ}$, corresponding to (002) and (101) of the pseudographitic domains, respectively [23]. The diffraction peaks of MC are broad and weak, indicating the limited graphitization degree. In addition, there are no obvious impurity peaks in the XRD pattern of MC, and the content of $\mathrm{Si}$ is 0.39 at $\%$ according to the XPS result. The above results indicate that the silica template was removed by $\mathrm{HF}$, and the MC was successfully prepared. An obvious diffraction peak appears at $2 \theta$ value of $45^{\circ}$ and $65^{\circ}$ on the patterns of nZVI@MC, which corresponds to the (100) and (200) direction of $\alpha-\mathrm{Fe}$, indicating that the nZVI was successfully loaded on the surface of MC by liquid phase reduction method [24]. The diffraction peak of S-nZVI@MC is sharper, indicating that sulfidation can enhance the crystallinity of nZVI. There is no peak of sulfur species $\left(\mathrm{FeS}\right.$ or $\left.\mathrm{FeS}_{2}\right)$ on the XRD pattern of S-nZVI@MC, which may be due to the low sulfur content or poor crystallinity of sulfur species [25]. After $3 \mathrm{~h}$ of reaction with TCP, the peak intensity of S-nZVI@MC has no obvious decrease. However, a weaker diffraction peak of $\mathrm{Fe}_{2} \mathrm{O}_{3}$ was observed, indicating that only part of the nZVI reacted with TCP. In addition, the reaction between such non-target reactants as $\mathrm{H}_{2} \mathrm{O}$ and nZVI should be effectively inhibited, which is attributed to the protection effect of FeS [25].

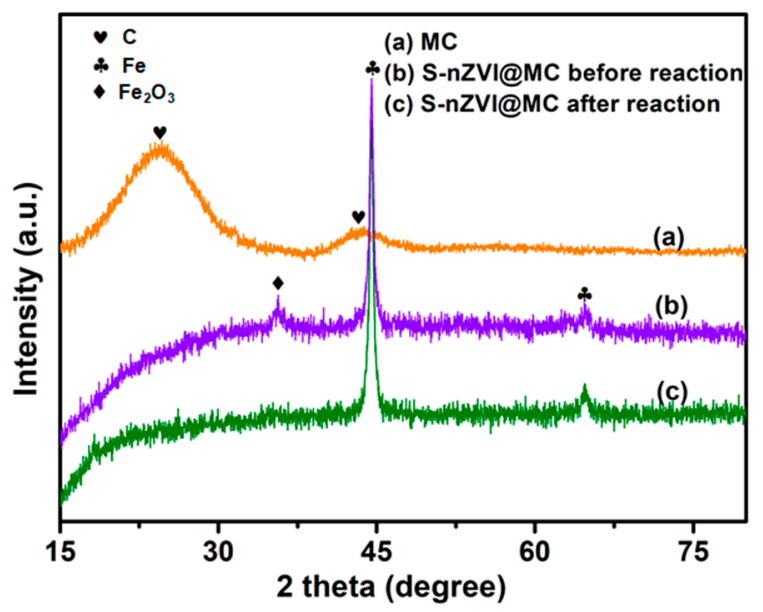

Figure 3. X-ray diffraction (XRD) patterns of MC, S-nZVI@MC before and after reaction. 
In order to further verify the formation of Fe and $S$ before and after the reaction with TCP, XPS spectra were further analyzed. The results (Figure $4 \mathrm{a}$ ) showed that the $\mathrm{S}$ spectrum can be divided into three peaks, which are located at $161.9 \mathrm{eV}, 163.8$, and $168.8 \mathrm{eV}$ assigned to $\mathrm{S}^{2-}, \mathrm{S}^{\mathrm{n}-}$ and $\mathrm{SO}_{4}{ }^{2-}$, respectively [26]. As calculated, the molar content of $\mathrm{S}^{2-}, \mathrm{S}^{\mathrm{n}-}$ and $\mathrm{SO}_{4}{ }^{2-}$ is $27.45 \%, 48.30 \%$ and $24.24 \%$. After the reaction with TCP, the peak intensity of $S^{2-}$ decreased obviously, accompany with the increase of $\mathrm{S}^{\mathrm{n}-}$ (Figure $4 \mathrm{~b}$ ). The $\mathrm{S}^{2-}$ molar content decreased to $12.43 \%$, and the molar content of $\mathrm{SO}_{4}{ }^{2-}$ increased to $55.31 \%$, which indicates that $S$ is oxidized after the reaction with TCP.
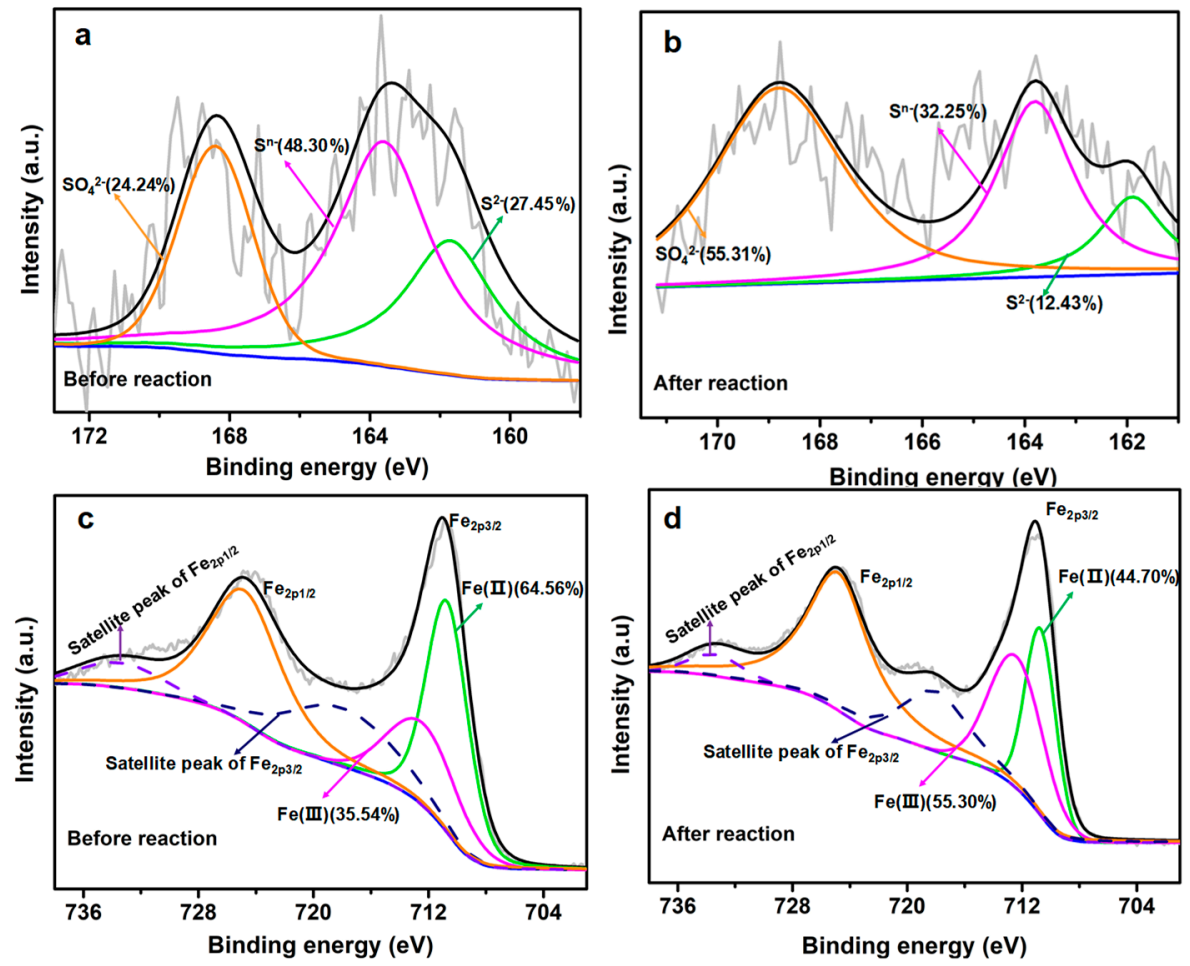

Figure 4. X-ray photoelectron spectroscopy (XPS) spectra of (a,b) S2p and (c,d) Fe2p region S-nZVI@MC before and after reaction.

The high-resolution XPS spectra of Fe $2 \mathrm{p}$ region was shown in Figure $4 \mathrm{c}$,d. The Fe spectrum can be divided into four peaks. The peaks at 710.7 and $712.4 \mathrm{eV}$ are assigned to $\mathrm{Fe}_{2 \mathrm{p} 3 / 2}$ for $\mathrm{Fe}$ (II) and Fe (III), respectively [26]. The peak located at $724.8 \mathrm{eV}$ related to $\mathrm{Fe}_{2 \mathrm{p} 1 / 2}$ for Fe (III). Besides, the satellite peak positions for $\mathrm{Fe}_{2 \mathrm{p} 1 / 2}$ and $\mathrm{Fe}_{2 \mathrm{p} 3 / 2}$ were 718 and $733.4 \mathrm{eV}$. Before reaction, two broad peaks at $724.8 \mathrm{eV}$ $\left(\mathrm{Fe}_{2 \mathrm{p} 1 / 2}\right)$ and $711 \mathrm{eV}\left(\mathrm{Fe}_{2 \mathrm{p} 2 / 3}\right)$ corresponding to iron oxides $\left(\mathrm{Fe}^{2+}\right.$ and $\left.\mathrm{Fe}^{3+}\right)$ are obvious (Figure $\left.4 \mathrm{c}\right)$, because a portion of $\mathrm{nZVI}$ could be oxidized after the reaction with $\mathrm{SO}_{4}{ }^{2-}$ during preparation or testing process, resulting in the formation of iron oxides or iron sulfides. However, it is worth noting that XPS can only detect a material surface of several nanometers' depth, and the $\mathrm{Fe}^{0}$ core may not be effectively detected [25]. The content of Fe (II) and Fe (III) is $64.56 \%$ and $35.54 \%$ before reaction. However, the $\mathrm{Fe}_{2 \mathrm{p} 3 / 2}$ peak for $\mathrm{Fe}$ (III) is strengthened, while the peak corresponding to Fe (II) is weakened (Figure $4 \mathrm{~d}$ ). The ratio of Fe (II) is decreased to $44.70 \%$, while Fe (III) is increased to $55.30 \%$, which indicates that Fe (0) and part of the Fe (II) in S-nZVI@MC were transformed to Fe (III) after reacting with TCP.

\subsection{Adsorption Performance}

The removal of TCP by nZVI before and after sulfidation was investigated. As shown in Figure 5a, the MC supporter has a certain adsorption capacity for TCP in water and the removal efficiency is about $30 \%$ after $120 \mathrm{~min}$, which can be ascribed to the pore-filling effect. The abundant mesopores can provide accessible channels for TCP molecular transport and adsorption. The removal efficiency of bare nZVI towards TCP was about 50\% due to the adsorption and reduction effects. It is noteworthy 
that the removal efficiency was significantly increased after sulfidation, which is increased to $91 \%$. The enhanced removal efficiency indicated that the sulfide modification greatly increases the reactivity of nZVI to TCP, because the -Cl groups on TCP have strong affinity with S-nZVI due to the hydrophobic effect of iron sulfide. Furthermore, the iron sulfide coated on the surface of nZVI can inhibit the formation of iron oxide, while iron sulfide with low-band gap can promote the rapid transfer of electrons from the nZVI core [27].

The adsorption kinetics of MC, nZVI and S-nZVI@MC were further investigated as the following equations:

$$
\begin{aligned}
& \text { pseudo-first kinetic: } \ln \left(\mathrm{q}_{\mathrm{e}}-\mathrm{q}_{\mathrm{t}}\right)=\ln \mathrm{q}_{\mathrm{e}}-k_{1} \mathrm{t} \\
& \text { pseudo-second kinetic: } \mathrm{t} / \mathrm{q}_{\mathrm{t}}=1 / k_{2} \mathrm{q}^{2}+\mathrm{t} / \mathrm{q}_{\mathrm{e}}
\end{aligned}
$$

where $\mathrm{q}_{\mathrm{e}}$ and $\mathrm{q}_{\mathrm{t}}\left(\mathrm{mg} \mathrm{g}^{-1}\right)$ was the removal capacity of TCP at time $\mathrm{t}(\mathrm{min})$ and equilibrium, respectively. $k_{1}\left(\mathrm{~min}^{-1}\right)$ and $k_{2}\left(\mathrm{~g} \mathrm{mg}^{-1} \mathrm{~min}^{-1}\right)$ was the equilibrium rate constant of both kinetic models $[28,29]$.

The kinetic fitting results were shown in Figure $5 b, c$ and the kinetics parameters are summarized in Table 1. On basic of the $\mathrm{R}^{2}$, the pseudo-second kinetic model can better describe the TCP removal behavior, suggesting that the TCP removal by nZVI and S-nZVI@MC is controlled by chemical processes, and the electronic exchange existed between TCP and S-nZVI@MC. Obviously, the rate constant $k_{2}$ of S-nZVI@MC was $0.0018 \mathrm{~g} \mathrm{mg}^{-1} \mathrm{~min}^{-1}$, higher than that of MC and nZVI (0.0012 and $0.0007 \mathrm{~g} \mathrm{mg}^{-1} \mathrm{~min}^{-1}$ ), which further reveals a higher affinity between S-nZVI@MC and TCP owing to the hydrophobic effect [28]. The highest $k$ of S-nZVI@MC further indicates that the sulfide treatment can increase the reactivity.
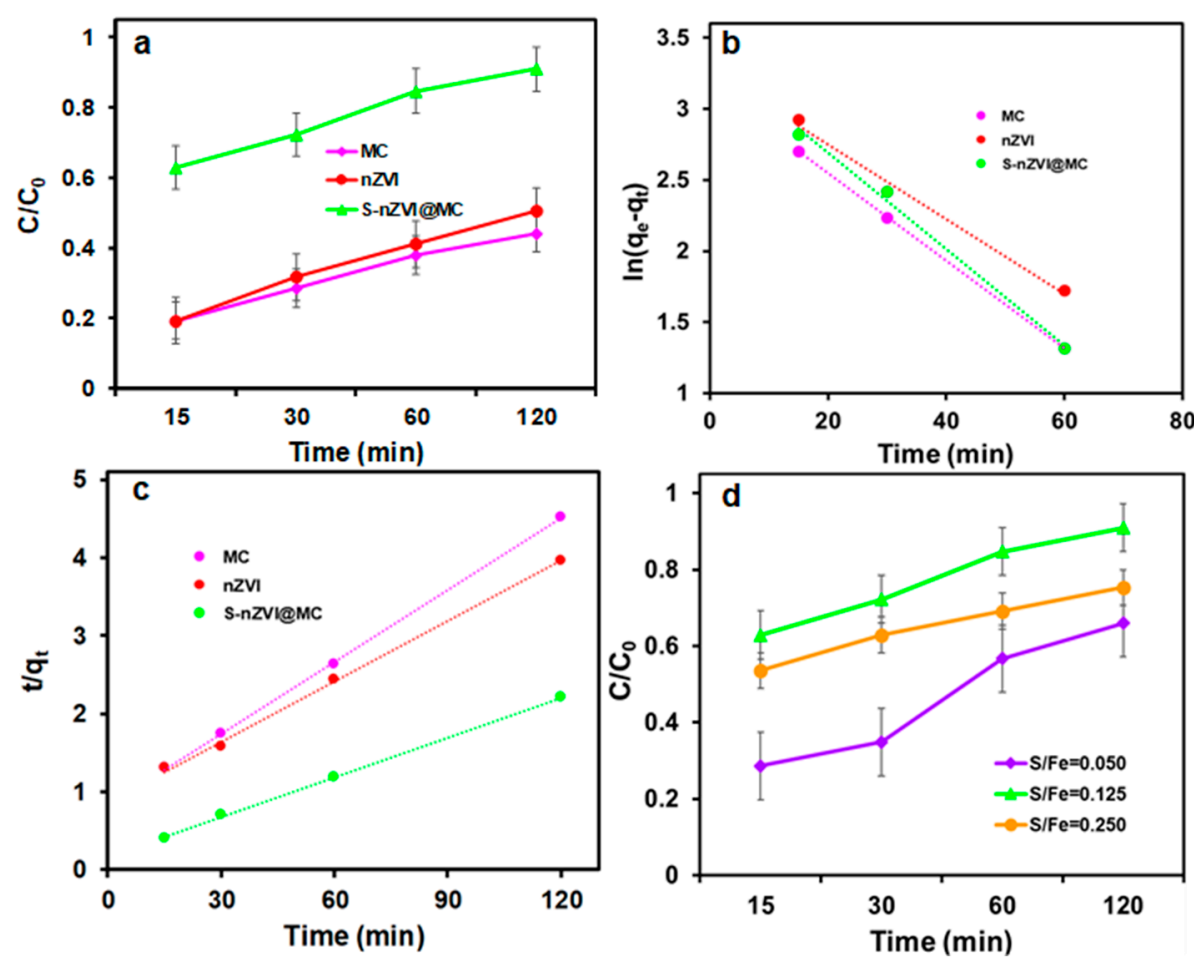

Figure 5. (a) The 2,4,6-trichlorphenol (TCP) removal performance, (b,c) kinetics study by MC, nZVI (d) S-nZVI@MC and the effect of Fe/S ratio on removal performance of TCP by S-nZVI@MC.

$\mathrm{Fe} / \mathrm{S}$ is one of the most critical factors that would influence the reactivity of S-nZVI@MC. The S-nZVI@MC particles sysnthesized at different S/Fe were further used for TCP removal, and the results are presented in Figure $5 \mathrm{~d}$. Obviously, the removal efficiency ranges from $60 \%$ to $90 \%$ depending on the different S/Fe ratio. However, the removal efficiency of S-nZVI@MC to TCP varies with the S/Fe ratio, for optimal S/Fe (0.125) the maximum removal efficiency can reach $90 \%$. With lower 
$\mathrm{S} / \mathrm{Fe}(0.05)$, the lower content of $\mathrm{S}^{2-}$ results in ineffective protection of nZVI, and thus the removal efficiency of TCP is lower. The removal efficiency decreased when the S/Fe further increased to 0.25 , because excessive iron sulfide, especially $\mathrm{FeS}_{2}$ with a higher band gap (0.95) generated could slow down electron transfer [27]. Moreover, the corrosion of zero-valent iron is inhibited at high S/Fe, leading to nZVI reactivity waste. Therefore, it is critical to optimize $\mathrm{S} / \mathrm{Fe}$ ratio during the sulfidation process, and the optimal S/Fe molar ratio is 0.125 was used in the following TCP removal experiments.

Table 1. Kinetic model parameters for the removal of 2,4,6-trichlorphenol (TCP) by MC, nZVI and S-nZVI@MC.

\begin{tabular}{|c|c|c|c|c|c|c|}
\hline \multirow[t]{2}{*}{ Sample } & \multicolumn{3}{|c|}{ Pseudo-First-Order-Model } & \multicolumn{3}{|c|}{ Pseudo-Second-Order-Model } \\
\hline & $k_{1}\left(\min ^{-1}\right)$ & $\mathrm{q}_{\mathrm{e}}\left(\mathrm{mg} \mathrm{g}^{-1}\right)$ & $\mathbf{r}^{2}$ & $k_{2}\left(\mathrm{~g} \mathrm{mg}^{-1} \min ^{-1}\right)$ & $\mathrm{q}_{\mathrm{e}}\left(\mathrm{mg} \mathrm{g}^{-1}\right)$ & $\mathbf{r}^{2}$ \\
\hline $\mathrm{MC}$ & 0.0308 & 23.64 & 1.000 & 0.0012 & 32.57 & 0.9998 \\
\hline nZVI & 0.0262 & 26.43 & 0.9894 & 0.0007 & 38.75 & 0.9985 \\
\hline S-nZVI@MC & 0.0339 & 29.14 & 0.9951 & 0.0018 & 58.82 & 0.9996 \\
\hline
\end{tabular}

As known, the $\mathrm{pH}$ value of aqueous solution has great effect on nZVI. In acidic conditions, nZVI could be rapidly corroded and tends to exhibit better activity. While in alkaline conditions, a large hydroxide passivation layer is formed on the surface of zero-valent iron, thereby inhibiting its activity. It has been demonstrated that sulfidation modification can promote electron transfer and inhibits surface passivation. However, the effect of sulfidation on nZVI reactivity under different $\mathrm{pH}$ conditions still needs further discussion. Herein, the removal efficiency of S-nZVI@MC under different pH value ranging from 4 to 11 was investigated. As shown in Figure 6a, the removal efficiency reached a maximum of $91 \%$ at $\mathrm{pH}$ of 4.0 , and then decreased to $82 \%$ when the $\mathrm{pH}$ value increased to 11.0 , which might be due to the formation of iron oxides on the surface of S-nZVI@BC [30].

The effect of S-nZVI@MC dosage was further investigated under the condition of $\mathrm{pH}=4.0$, $30 \mathrm{mg} \mathrm{L}^{-1} \mathrm{TCP}$ aqueous solution and the results are shown in Figure $6 \mathrm{~b}$. The removal efficiency of TCP was enhanced when the dosage was increased. Accordingly, the removal efficiency is $57 \%, 69 \%$, $88 \%, 91 \%, 97 \%$ and $100 \%$ for $0.1,0.3,0.5,1.0,2.0$ and $3.0 \mathrm{~g} \mathrm{~L}^{-1}$, respectively. When the dosage is high, there are more surface reactive and adsorptive sites in the reaction system, so the removal rate of TCP is higher. However, an excessive dosage is likely to cause agglomeration of materials and low utilization of reaction sites. Hence, the dosage of the further study is $0.5 \mathrm{~g} \mathrm{~L}^{-1}$ for TCP removal.
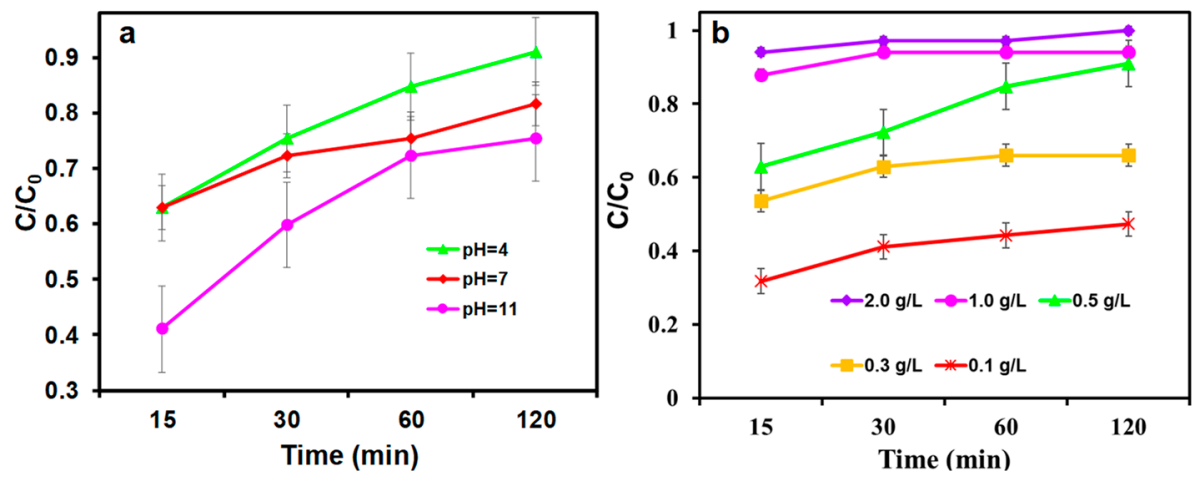

Figure 6. (a) Initial pH and (b) dosage on removal performance of TCP by S-nZVI@MC.

Figure 7a shows the removal efficiency of S-nZVI@MC towards TCP under different initial concentrations ranging from 30 to $100 \mathrm{mg} \mathrm{L}^{-1}$. Generally, the TCP removal efficiency is decreased when the initial concentration increased. As the initial concentration increased from 30 to $100 \mathrm{mg} \mathrm{L}^{-1}$, the removal efficiency decreased from $91 \%$ to $32 \%$. With higher initial concentration, the surface of S-nZVI@MC is easier to passivate, and thus the diffusion of contaminants and the electron transfer between nZVI and contaminants are inhibited, resulting in a reduced contaminants removal efficiency. 
Besides, at high concentrations, a large number of target contaminant molecules compete for limited reaction sites on the surface of the S-nZVI@MC, which also leads to reduced TCP removal efficiency [31]. The adsorption isotherm was used to analyze the interactions between TCP and S-nZVI@MC, which further can be fitted by Langmuir and Freundlich models. As shown in Figure 7b, the TCP removal profile can be described well by the Langmuir model, which has a higher correlation coefficient $\mathrm{R}^{2}$ (0.993). In contrast, the Freundlich model has a relative lower $\mathrm{R}^{2}(0.936)$. Hence, the adsorption of TCP on the surface of S-nZVI@MC has been dominated by monolayer adsorption [32].
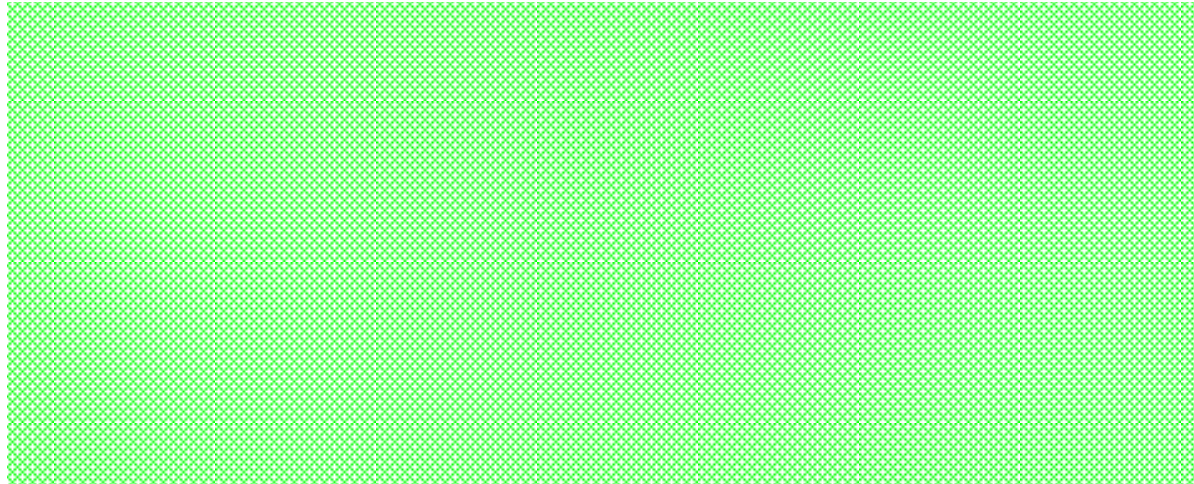

Figure 7. (a) Effect of initial concentration on removal performance and (b) the linear Langmuir isotherm plot for TCP on S-nZVI@MC.

Reusability is another critical property for the application of nZVI-based material. The reusability of S-nZVI@MC was further tested in $30 \mathrm{mg} \mathrm{L}^{-1} \mathrm{TCP}$ solution with the dose of $0.5 \mathrm{~g} \mathrm{~L}^{-1}$ and reaction time of 3 h. As shown in Figure 8, the stability of S-nZVI@MC was evaluated by reactions 5 times. The reaction ability has no significant decline after five repeated experiments, indicating there was no significant passivation on the surface of S-nZVI@MC. The good stability can be ascribed to effective sulfidation. Hence, the results of reusability experiments further demonstrate that S-nZVI@MC can be practically applied to pollutant adsorption and reduction.

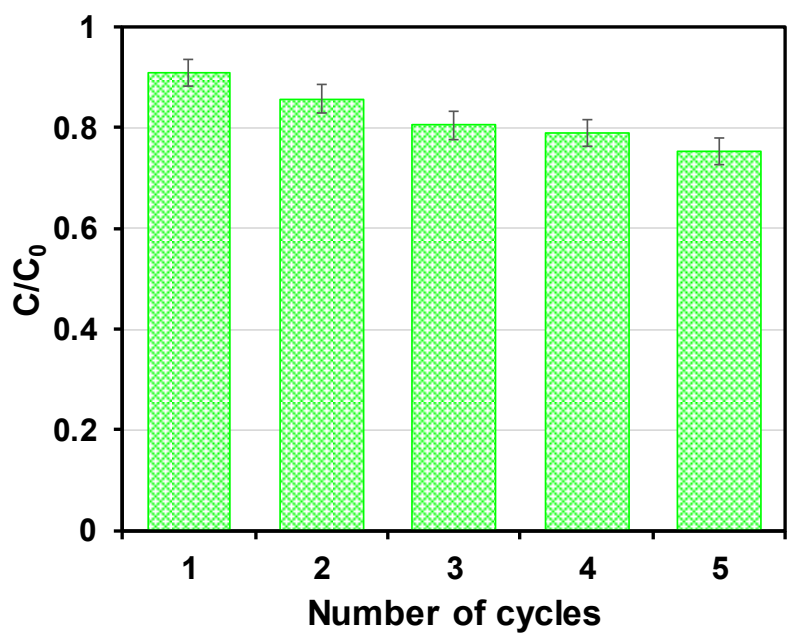

Figure 8. The reuse ability of S-nZVI@MC towards TCP.

\section{Conclusions}

In summary, biomass-derived MC-loaded S-nZVI was successfully prepared for effective TCP removal. MC was selected as the support for $\mathrm{nZVI}$, because its mesoporous structure was beneficial to nZVI dispersion and mass transfer. Moreover, MC prepared from biomass waste was a cost-effective material for practical application. Further surface sulfidation improved the antioxidation of nZVI due 
to the protection effect of FeS shell. SEM, TEM, XRD and XPS measurements demonstrated that the nZVI particles were successfully loaded on the MC supporter and the FeS was well coated on the surface the nZVI. Furthermore, the aggregation of nZVI and the iron oxide formation was prevented according the structure characterization. S-nZVI@MC was most effective for TCP removal as compared to $\mathrm{MC}$ and $\mathrm{nZVI}$, and the removal efficiency reached the highest value at a ratio of $\mathrm{S} / \mathrm{Fe}=0.125$. The effect of ambient conditions such as solution $\mathrm{pH}$, initial concentration and dosage on TCP removal were also studied, and results revealed that the low solution $\mathrm{pH}$ value and concentration and higher dosage had positive effect on TCP removal. Furthermore, the removal behavior was fitted with a Langmuir model and pseudo-second kinetic model. The removal mechanism of S-nZVI@MC towards TCP could by analyzed by fast adsorption and effective reduction by S-nZVI. Therefore, the synergistic effect of MC loading and surface sulfide-modification promoted high removal efficiency of S-nZVI@MC towards TCP.

Author Contributions: Conceptualization, H.W., S.C. and J.Y.; Methodology, M.Z. and N.L.; Validation, L.S.; Formal Analysis, L.S.; Investigation, L.S.; Resources, H.W.; Data Curation, H.W. and L.S.; Writing-Original Draft Preparation, H.W.; Writing-Review and Editing, S.C. and J.Y.; Visualization, G.Q.; Supervision, J.Y.; Project Administration, H.W.; Funding Acquisition, H.W.

Funding: This research was funded by the Natural Science Foundation of Jiangsu Province (BK20170475) and Joint Open Fund of Jiangsu Collaborative Innovation Center for Ecological Building Material and Enviromental Protection Equipments and Key Laboratory for Advanced Technology in Environmental Protection of Jiangsu Province.

Acknowledgments: The authors would like to thank C.J.M. from Analysis and Test Center of Yancheng Institute of Technology, for help with the TEM measurements.

Conflicts of Interest: The authors declare no conflict of interest

\section{References}

1. Garba, Z.N.; Zhou, W.; Lawan, I.; Xiao, W.; Zhang, M.; Wang, L.; Chen, L.; Yuan, Z. An overview of chlorophenols as contaminants and their removal from wastewater by adsorption: A review. J. Environ. Manag. 2019, 241, 59-75. [CrossRef] [PubMed]

2. Olu-Owolabi, B.I.; Alabi, A.H.; Diagboya, P.N.; Unuabonah, E.I.; During, R.A. Adsorptive removal of 2,4,6-trichlorophenol in aqueous solution using calcined kaolinite-biomass composites. J. Environ. Manag. 2017, 192, 94-99. [CrossRef] [PubMed]

3. Han, A.; Zhang, H.; Lu, D.; Sun, J.; Chuah, G.K.; Jaenicke, S. Efficient photodegradation of chlorophenols by $\mathrm{BiOBr} / \mathrm{NaBiO} 3$ heterojunctioned composites under visible light. J. Hazard. Mater. 2018, 341, 83-92. [CrossRef] [PubMed]

4. Shu, X.; Yang, Q.; Yao, F.; Zhong, Y.; Ren, W.; Chen, F.; Sun, J.; Ma, Y.; Fu, Z.; Wang, D.; et al. Electrocatalytic hydrodechlorination of 4-chlorophenol on Pd supported multi-walled carbon nanotubes particle electrodes. Chem. Eng. J. 2019, 358, 903-911. [CrossRef]

5. Chen, C.; Geng, X.; Huang, W. Adsorption of 4-chlorophenol and aniline by nanosized activated carbons. Chem. Eng. J. 2017, 327, 941-952. [CrossRef]

6. Zou, Y.; Wang, X.; Khan, A.; Wang, P.; Liu, Y.; Alsaedi, A.; Hayat, T.; Wang, X. Environmental remediation and application of nanoscale zero-valent iron and its composites for the removal of heavy metal ions: A Review. Environ. Sci. Technol. 2016, 50, 7290-7304. [CrossRef]

7. Zhao, X.; Liu, W.; Cai, Z.; Han, B.; Qian, T.; Zhao, D. An overview of preparation and applications of stabilized zero-valent iron nanoparticles for soil and groundwater remediation. Water Res. 2016, 100, 245-266. [CrossRef]

8. Shi, X.; Ruan, W.; Hu, J.; Fan, M.; Cao, R.; Wei, X. Optimizing the removal of rhodamine B in aqueous solutions by reduced graphene oxide-supported nanoscale zerovalent iron (nZVI/rGO) using an artificial neural network-genetic algorithm (ANN-GA). Nanomaterials 2017, 7, 134. [CrossRef]

9. Li, J.; Chen, C.L.; Zhu, K.R.; Wang, X.K. Nanoscale zero-valent iron particles modified on reduced graphene oxides using a plasma technique for Cd(II) removal. J. Taiwan Inst. Chem. Eng. 2016, 59, 389-394. [CrossRef] 
10. Teng, W.; Fan, J.; Wang, W.; Bai, N.; Liu, R.; Liu, Y.; Deng, Y.; Kong, B.; Yang, J.; Zhao, D.; et al. Nanoscale zero-valent iron in mesoporous carbon (nZVI@C): Stable nanoparticles for metal extraction and catalysis. J. Mater. Chem. A 2017, 5, 4478-4485. [CrossRef]

11. Bhattacharjee, S.; Ghoshal, S. Sulfidation of nanoscale zerovalent iron in the presence of two organic macromolecules and its effects on trichloroethene degradation. Environ. Sci-Nano 2018, 5, 782-791. [CrossRef]

12. Li, H.; Qiu, Y.F.; Wang, X.L.; Yang, J.; Yu, Y.J.; Chen, Y.Q.; Liu, Y.D. Biochar supported Ni/Fe bimetallic nanoparticles to remove 1,1,1-trichloroethane under various reaction conditions. Chemosphere 2017, 169, 534-541. [CrossRef] [PubMed]

13. Dai, Y.; Hu, Y.; Jiang, B.; Zou, J.; Tian, G.; Fu, H. Carbothermal synthesis of ordered mesoporous carbon-supported nano zero-valent iron with enhanced stability and activity for hexavalent chromium reduction. J. Hazard. Mater. 2016, 309, 249-258. [CrossRef] [PubMed]

14. Khalil, A.M.E.; Eljamal, O.; Amen, T.W.M.; Sugihara, Y.; Matsunaga, N. Optimized nano-scale zero-valent iron supported on treated activated carbon for enhanced nitrate and phosphate removal from water. Chem. Eng. J. 2017, 309, 349-365. [CrossRef]

15. Wu, X.; Yang, Q.; Xu, D.; Zhong, Y.; Luo, K.; Li, X.; Chen, H.; Zeng, G. Simultaneous Adsorption/Reduction of Bromate by Nanoscale Zerovalent Iron Supported on Modified Activated Carbon. Ind. Eng. Chem. Res. 2013, 52, 12574-12581. [CrossRef]

16. Ling, X.F.; Li, J.S.; Zhu, W.; Zhu, Y.Y.; Sun, X.Y.; Shen, J.Y.; Han, W.Q.; Wang, L.J. Synthesis of nanoscale zero-valent iron/ordered mesoporous carbon for adsorption and synergistic reduction of nitrobenzene. Chemosphere 2012, 87, 655-660. [CrossRef]

17. Fan, D.; O'Brien Johnson, G.; Tratnyek, P.G.; Johnson, R.L. Sulfidation of nano zerovalent iron (nZVI) for improved selectivity during in-situ chemical reduction (ISCR). Environ. Sci. Technol. 2016, 50, 9558-9565. [CrossRef]

18. Xu, J.; Wang, Y.; Weng, C.; Bai, W.; Jiao, Y.; Kaegi, R.; Lowry, G.V. Reactivity, selectivity, and long-term performance of sulfidized nanoscale zerovalent iron with different properties. Environ. Sci. Technol. 2019, 53, 5936-5945. [CrossRef]

19. Xu, J.; Cao, Z.; Wang, Y.; Zhang, Y.; Gao, X.; Ahmed, M.B.; Zhang, J.; Yang, Y.; Zhou, J.L.; Lowry, G.V. Distributing sulfidized nanoscale zerovalent iron onto phosphorus-functionalized biochar for enhanced removal of antibiotic florfenicol. Chem. Eng. J. 2019, 359, 713-722. [CrossRef]

20. Li, J.; Zhang, X.; Sun, Y.; Liang, L.; Pan, B.; Zhang, W.; Guan, X. Advances in Sulfidation of Zerovalent Iron for Water Decontamination. Environ. Sci. Technol. 2017, 51, 13533-13544. [CrossRef]

21. Gao, J.; Yang, L.; Liu, Y.; Shao, F.; Liao, Q.; Shang, J. Scavenging of Cr(VI) from aqueous solutions by sulfide-modified nanoscale zero-valent iron supported by biochar. J. Taiwan Inst. Chem. Eng. 2018, 91, 449-456. [CrossRef]

22. He, F.; Li, Z.J.; Shi, S.S.; Xu, W.Q.; Sheng, H.Z.; Gu, Y.W.; Jiang, Y.H.; Xi, B.D. Dechlorination of excess trichloroethene by bimetallic and sulfidated nanoscale zero-valent iron. Environ. Sci. Technol. 2018, 52, 8627-8637. [CrossRef] [PubMed]

23. Wang, H.; Zhang, X.; Wang, Y.; Quan, G.; Han, X.; Yan, J. Facile synthesis of magnetic nitrogen-doped porous carbon from bimetallic metal-organic frameworks for efficient norfloxacin removal. Nanomaterials 2018, 8 , 664. [CrossRef] [PubMed]

24. Xue, W.; Huang, D.; Zeng, G.; Wan, J.; Zhang, C.; Xu, R.; Cheng, M.; Deng, R. Nanoscale zero-valent iron coated with rhamnolipid as an effective stabilizer for immobilization of $\mathrm{Cd}$ and $\mathrm{Pb}$ in river sediments. J. Hazard. Mater. 2018, 341, 381-389. [CrossRef]

25. Cao, Z.; Liu, X.; Xu, J.; Zhang, J.; Yang, Y.; Zhou, J.; Xu, X.; Lowry, G.V. Removal of antibiotic florfenicol by sulfide-modified nanoscale zero-valent iron. Environ. Sci. Technol. 2017, 51, 11269-11277. [CrossRef]

26. Zhang, D.; Li, Y.; Tong, S.; Jiang, X.; Wang, L.; Sun, X.; Li, J.; Liu, X.; Shen, J. Biochar supported sulfide-modified nanoscale zero-valent iron for the reduction of nitrobenzene. RSC Adv. 2018, 8, 22161-22168. [CrossRef]

27. Dong, H.R.; Zhang, C.; Deng, J.M.; Jiang, Z.M.; Zhang, L.H.; Cheng, Y.J.; Hou, K.J.; Tang, L.; Zeng, G.M. Factors influencing degradation of trichloroethylene by sulfide-modified nanoscale zero-valent iron in aqueous solution. Water Res. 2018, 135, 1-10. [CrossRef] 
28. Wang, Z.; Yang, J.; Li, Y.; Zhuang, Q.; Gu, J. In situ carbothermal synthesis of nanoscale zero-valent iron functionalized porous carbon from metal-organic frameworks for efficient detoxification of chromium(VI). Chem. Eur. J. 2018, 2018, 23-30. [CrossRef]

29. Pocoví-Martínez, S.; Cassano, D.; Voliani, V. Naked nanoparticles in silica nanocapsules: A versatile family of nanorattle catalysts. ACS Appl. Nano Mater. 2018, 1, 1836-1840. [CrossRef]

30. Li, D.; Zhu, X.; Zhong, Y.; Huang, W.; Peng, P. Abiotic transformation of hexabromocyclododecane by sulfidated nanoscale zerovalent iron: Kinetics, mechanism and influencing factors. Water Res. 2017, 121, 140-149. [CrossRef]

31. Dong, H.; Jiang, Z.; Zhang, C.; Deng, J.; Hou, K.; Cheng, Y.; Zhang, L.; Zeng, G. Removal of tetracycline by $\mathrm{Fe} / \mathrm{Ni}$ bimetallic nanoparticles in aqueous solution. J. Colloid Interface Sci. 2018, 513, 117-125. [CrossRef] [PubMed]

32. Yang, F.; Zhang, S.; Sun, Y.; Cheng, K.; Li, J.; Tsang, D.C.W. Fabrication and characterization of hydrophilic corn stalk biochar-supported nanoscale zero-valent iron composites for efficient metal removal. Bioresource Technol. 2018, 265, 490-497. [CrossRef] [PubMed]

(C) 2019 by the authors. Licensee MDPI, Basel, Switzerland. This article is an open access article distributed under the terms and conditions of the Creative Commons Attribution (CC BY) license (http://creativecommons.org/licenses/by/4.0/). 